experience with multiple myeloma in the patients aged more than 60 years.

Objectives This is a retrospective study of 300 newly diagnosed multiple myeloma patients above 60 years of age treated in the Department of Medical Oncology, Regional Cancer Center, Thiruvananthapuram, Kerala, India, during the period between 2014 and 2017. The medical records of the patients were studied and following data were collected: demographic and clinical details, diagnostic and staging workup, primary treatment, response assessment, relapse, and survival. Survival was estimated using the Kaplan-Meier method.

Results A total of 300 patients were included in the study. The median age was 66 years with a male-to-female ratio of 1.4:1. The common clinical presentations were backache (134), fatigue (49), lower respiratory infection (20), and paraparesis (14). Monoclonal protein was immunoglobulin (Ig)- $\mathrm{G}$ in 199 patients (66.6\%), IgA in 52 patients (17.4\%), IgM in 2 patients, and IgD in 1 patient. Light-chain disease was seen in 42 patients (14\%). One hundred and sixty patients $(53.5 \%)$ had ISS stage III. Only 285 patients received treatment, of which 203 (67.8\%) received bortezomib-based regimen, - bortezomib and dexamethasone (BD; 33.4\%); bortezomib, lenalidomide, and dexamethasone (BLD; 19.7\%); bortezomib, cyclophosphamide, and dexamethasone (VCD; 8.7\%); bortezomib, thalidomide, and dexamethasone (BTD; 2.3\%); and bortezomib, melphalan, and prednisolone (3.7\%). Nonbortezomib-based regimens used were melphalan and prednisolone (MP) alone or with thalidomide or lenalidomide (15\%), lenalidomide and dexamethasone (LD; $10.4 \%$ ), and thalidomide and dexamethasone (TD; 2\%). Response assessment was done as per IMWG guidelines. Fifty-seven (26.3\%) patients achieved complete response (CR), 94 (43.3\%) achieved very good partial response (VGPR), 19 (8.8\%) attained partial response (PR), $15(5.6 \%)$ had stable disease, and 46 (15.4\%) developed progressive disease. With bortezomib-based regimens, 119 patients (58.3\%) achieved CR/VGPR, and with non-bortezomib based regimens, 42 patients (51.2\%) achieved CR/VGPR. One hundred and forty-three patients (47.8\%) received maintenance therapy of which 79 received maintenance with bortezomib, 49 with lenalidomide, and 15 with thalidomide. The average duration of maintenance was 24 months. Second-line chemotherapy regimens were used in 37 patients. Agents used were MP, LD, TD, and VCD. With second-line treatment, 15 patients achieved VGPR, 10 patients achieved partial response, and 25 patients developed progressive disease. Third-line chemotherapy regimens were used in 22 patients and the regimens used were pomalidomide and dexamethasone, MP, TD, LD, vincristine, doxorubicin, and dexamethasone and carfilzomib and dexamethasone. At a median follow-up of 34 months, the 2 -year overall survival (OS) was $68 \%$. The median progression-free survival was 21 months. The 2 -year OS for patients receiving initial bortezomib-based regimen was $67.8 \%$ and non-bortezomib based regimen was $68 \%$ which was similar.

Conclusion In this study, CR/VGPR rates and 2-year OS in patients treated with bortezomib and non-bortezomib based regimens were not statistically significant.

Keywords: multiple myeloma, elderly, treatment outcome, regional cancer center, India

\section{Epithelial Ovarian Cancer: Real-World Outcomes}

Thejeswar Nakka', Prasanth Ganesan'1, Luxitaa Goenka', Biswajit Dubashi', Smita Kayal', Latha Chaturvedula ${ }^{2}$, Dasari Papa ${ }^{2}$, Prasanth Penumadu ${ }^{3}$, Narendran Krishnamoorthy' ${ }^{1}$, Divya B. Thumaty ${ }^{1}$

'Department of Medical Oncology, JIPMER, Puducherry

2Department of Obstetrics and Gynaecology, JIPMER, Puducherry

${ }^{3}$ Department of Surgical Oncology, JIPMER, Puducherry

\section{Address for correspondence:}

Dr Prasanth Ganesan, MD, DM, Additional Professor

and Head, Department of Medical Oncology,

JIPMER, Puducherry 605006, India (e-mail: pg1980@gmail.com)

\section{Abstract}

Introduction Ovarian cancer is the third most common cancer and the second most common cause of death among gynecological cancers in Indian women. Ovarian cancer is heterogeneous, among them, epithelial ovarian cancer (EOC) is the most common. Primary cytoreductive surgery along with six to eight cycles of a combination of platinum and taxanes chemotherapy is the cornerstone of first-line treatment in EOC.
This study was done to find clinicopathological factors affecting survival outcomes with first-line therapy in EOC in a real-world setting.

Objectives This study was aimed to find factors affecting progression-free survival (PFS) and overall survival (OS) with first-line treatment in EOC.

Materials and Methods We conducted a single-center retrospective study. We screened all the patients diagnosed with ovarian cancer from January 2015 till December 2019. We locked data in August 2019. Eligible patients were histologically confirmed EOC who underwent primary cytoreduction or received more than or equal to two cycles of chemotherapy or both. Patients who had received first-line treatment at another hospital were excluded.

Results Patients demographics and clinical characteristics: between January 5, 2015 to August 31, 2019, 435 patients with a diagnosis of ovarian malignancy were registered at our center. Among them, 406 (82\%) had EOC, 290 (64\%) newly diagnosed, and fulfilling eligibility criteria were included in the final analysis. The median age of the cohort was 53 years (range: $21-89$ years) and 157 patients (54\%) were >50 years of age (the Eastern Oncology Cooperative Group Performance status was $\geq 2$ in 124 patients [43\%]; median duration of symptoms was 3 months; and stage III/IV: 240 [83\%]). Grading of the tumor was available in 240 patients of which 219 (91\%) were of high grade. Subtyping was available in 272 patients (94\%) of which the serous subtype was the most common constituting 228 patients (79\%).

Treatment Most patients received chemotherapy $(n=283$ [98\%]) as the first modality of treatment (neoadjuvant/adjuvant and palliative). As neoadjuvant (NACT) in 130 patients (45\%) and as adjuvant following surgery in 81 patients (29\%). The most common chemotherapy regimen was a combination of carboplatin and paclitaxel in 256 patients (88\%). Among 290 patients 218 (75\%) underwent cytoreductive surgery. Among them, optimal cytoreduction was achieved in 108 patients (52\%). Optimal cytoreduction rate (OCR) with upfront surgery and after NACT was 44 and 53\%, respectively (Chi-square test: $0.86 ; p=0.35$ ).

Survival The median follow-up of the study was 17 months (range: 10-28 months) and it was 20 months (range: $12-35$ months) for patients who were alive. At last, follow-up, 149 patients $(51 \%)$ had progressed and 109 (38\%) died. The estimated median PFS and OS were 19 months (95\% Cl: $16.1-21.0)$ and 39 months (95\% Cl: 29.0-48.8), respectively. On multivariate analysis, primary surgery (HR: $0.1,95 \% \mathrm{Cl}: 0.06-0.21$; $p$-value: $<0.001$ ) and early-stage disease (HR: $0.2,95 \% \mathrm{Cl}: 0.1-0.6$; $p$-value 0.04 ) were associated with superior PFS and primary surgery (HR: $0.1,95 \% \mathrm{Cl}$ : $0.09-0.2$; $p$-value: $<0.001$ ) was associated with superior OS.

Conclusion Primary surgery (upfront or interval) was associated with improved survival. Newer agents like bevacizumab, poly-ADP (adenosine diphosphate)-ribose polymerase inhibitors and HIPEC should be incorporated precisely into first line of therapy to improve outcomes. Keywords: epithelial ovarian cancer, progression-free survival, overall survival

\section{To Predict Success of Postapheresis Yield and Post-Autologous Transplant Engraftment Based on Preapheresis Peripheral Blood CD34+ Cell Counts: An Indian Scenario-Based Study}

Pinki Devi ${ }^{1}$, Ganapathi Bhat ${ }^{2}$, Harish S. Ahuja ${ }^{3}$

'Department of Pathology, Jaslok Hospital and Research Center, Mumbai, Maharashtra, India

2Department of Medical Oncology and Stem Cell Transplant, Jaslok Hospital and Research Center, Mumbai, Maharashtra, India

${ }^{3}$ Department of Pathology, Blood Transfusion and Immunohematology, Jaslok Hospital and Research Center, Mumbai, Maharashtra, India

Address for correspondence: Pinki Devi, House No. 27, Sector 2, Rohtak, Haryana, India-124001 (e-mail: drpinkitmr@gmail.com).

\section{Abstract}

Introduction The use of hematopoietic stem cells for autologous and allogeneic transplantation has increased in the recent past significantly, due to introduction of newer chemotherapeutic drugs, immunological techniques, and better stem cell technology. Among the bone marrow and peripheral blood stem cells, collection of the latter being more convenient to the patient and associated with faster granulocyte and 